\title{
Divalent europium as a paramagnetic probe to study strontium precipitates in monocrystalline sodium chloride
}

Cite as: J. Chem. Phys. 77, 189 (1982); https://doi.org/10.1063/1.443640

Published Online: 31 August 1998

H. Murrieta S., F. J. López, J. García-Solé, M. Aguilar G., and J. Rubio O.

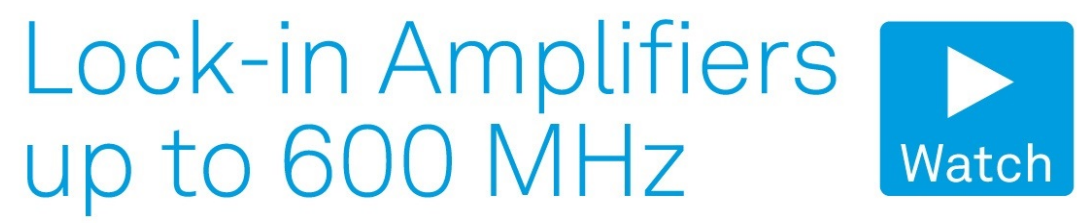




\title{
Divalent europium as a paramagnetic probe to study strontium precipitates in monocrystalline sodium chloride $e^{\text {a) }}$
}

\author{
H. Murrieta S., () F. J. López, J. Garcla-Solé, and M. Aguilar G. \\ Departamento de Optica y Estructura de la Materia, Universidad Autónoma de Madrid, Spain
}

J. Rubio O.

Instituto de Física, UNAM, Apartado Postal 20-364, Delegación Alvaro Obregón, 01000 México, D.F. (Received 21 December 1981; accepted 2 March 1982)

\begin{abstract}
Precipitation of divalent strontium ions in $\mathrm{NaCl}$ has been investigated using $\mathrm{Eu}^{2+}$ as a paramagnetic probe. The results presented in this paper show that the annealing of the quenched samples at $200^{\circ} \mathrm{C}$ produces the formation of the precipitated dihalide phase $\mathrm{SrCl}_{2}$ in the $\mathrm{NaCl}$ matrix. The EPR data established that the $\mathrm{Eu}^{2+}$ ions are located inside this precipitated structure and that they are substituting the host cation $\mathrm{Sr}^{2+}$ ions. However, the observed EPR spectrum shows a significant difference with respect to that reported for the same impurity ion in $\mathrm{SrCl}_{2}$ single crystals, i.e., the value for the cubic spin-Hamiltonian parameter $b_{4}^{0}$ is larger and its magnitude seems to decrease as the particle size of the precipitate increases. On the other hand, annealing of the quenched samples at room temperature produces another precipitated phase. In this case, the $\mathrm{EPR}$ data reveal that the $\mathrm{Eu}^{2+}$ ions also occupy a cubic symmetry site in this precipitated structure. However, the value for the $b_{4}^{0}$ parameter describing this cubic site is considerably smaller than the one measured for $\mathrm{Eu}^{2+}$ in $\mathrm{SrCl}_{2}$. Finally, an EPR spectrum, observed in the fast quenched samples, possessing axial symmetry and probably due to $\mathrm{Sr}^{2+}-\mathrm{Eu}^{2+}$-vacancy complexes is presented. Values for the spin-Hamiltonian parameters describing this new spectrum are also reported.
\end{abstract}

\section{INTRODUCTION}

It is well known that incorporation of doubly charged impurity ions $\left(\mathrm{M}^{2+}\right)$ into alkali halide single crystals produces an excess positive charge which is usually compensated by cation vacancies. The divalent cations and cation vacancies may form various kinds of complexes, depending upon the thermal treatment given to the crystal. Their structures have usually been investigated using optical and Electron Paramagnetic Resonance (EPR) techniques. When the impurity concentration is greater than the solubility limit, the divalent impurities leave the solid solution, building up microprecipitates in the crystal which have been studied by $x$-ray diffraction spectra, electron microscopy measurements, and light scattering, as well as by the use of the Mössbauer spectroscopy.

Although the isothermal decay of $\mathrm{M}^{2+}$ cation vacancy dipoles as a function of time have been extensively studied for a large number of different divalent impurity ions in $\mathrm{NaCl}$ and $\mathrm{KCl}$, there is still substantial controversy as to whether the initial aggregation product is a dimer or a trimer. ${ }^{1-3}$ Taking advantage of the fact that the EPR spectrum of a paramagnetic ion is quite sensitive to its local environment, Ikeya and Crawford were the first to employ a paramagnetic probe $\left(\mathrm{Mn}^{2+}\right)$ to analyze the structure of several aggregation products in $\mathrm{NaCl}$ doped with $\mathrm{Mg}^{2+}, \mathrm{Pb}^{2+}, \mathrm{Ca}^{2+}$, and $\mathrm{Ba}^{2+}$. $\mathrm{Al}-$ though their study was not conclusive with respect to the initial aggregation product, their results suggested the formation of dimers with $\langle 100\rangle$ symmetry, large aggregates in $\{111\}$ planes and $\mathrm{Mn}^{2+}$ ions occupying cubic

\footnotetext{
${ }^{2}$ Work partially supported by CONACYT (México) and CSIC (Spain) under contract ICAIESP-800666.

b) On leave from Instituto de Fisica, UNAM, México 01000, D.F.
}

symmetry sites in a second-phase precipitate in the samples analyzed.

Recently, Rappaz et al. ${ }^{5}$ have used the $\mathrm{Gd}^{3+}$ ion as a paramagnetic probe to investigate the crystal field effects induced by the size of particles of the dielectric material $\mathrm{SrCl}_{2}$. One of the most interesting results of this investigation is that the magnitude of the cubic spin-Hamiltonian parameter $b_{4}^{0}$ corresponding to the EPR spectrum of $\mathrm{Gd}^{3+}$ in a small particle sample $(<200$

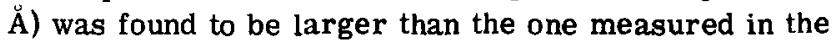
macro-single crystal by $\sim 1.6 \%$. This fact was interpreted as a consequence of the small particle size which produces a diminution in the host lattice parameter.

Ih the present paper, an investigation dealing with the precipitation of $\mathrm{Sr}^{2+}$ ions in the $\mathrm{NaCl}$ matrix is reported using $\mathrm{Eu}^{2+}$ as a paramagnetic probe. In order to avoid the possibility that the precipitation product contains several $\mathrm{Eu}^{2+}$ ions and that the exchange and dipole-dipole interactions between adjacent paramagnetic ions will result in the excessive broadening of the EPR lines which could obscure the fine structure splitting, a small amount of $\mathrm{Eu}^{2+}$ was employed in comparison with that of strontium. It should be pointed out, that the system $\mathrm{NaCl}-\mathrm{SrCl}_{2}$ was selected to perform this investigation, in view of the fact that additional information regarding the structure of the precipitated phases of $\mathrm{Sr}^{2+}$ in $\mathrm{NaCl}$ had previously been determined using $x$-ray diffraction and electron microscopy measurements. ${ }^{6-8}$ This fact allowed us to compare those studies with the EPR data presented here.

\section{EXPERIMENTAL}

Single crystals of $\mathrm{NaCl}$ doubly doped with $\mathrm{Sr}^{2+}$ and $\mathrm{Eu}^{2+}$ were grown from the melt by the Czochralski method under a controlled atmosphere of dry argon, the crys- 
tals being slowly cooled to room temperature in the furnace. The starting material was suprapure $\mathrm{NaCl}$ obtained from Merck. The dopants were $\mathrm{SrCl}_{2}$ and $\mathrm{EuCl}_{2}$, the latter being previously reduced from $\mathrm{EuCl}_{3} \cdot 6 \mathrm{H}_{2} \mathrm{O}$ using standard techniques. ${ }^{9}$ The concentration of the impurities in the crystals employed were determined by atomic absorption spectrophotometry and the results were $860 \mathrm{ppmm}$ (atomic) of $\mathrm{Sr}^{2+}$ and 8 ppmm of $\mathrm{Eu}^{2+}$. The latter concentration was also verified using the optical absorption spectrum of the doped samples following the same procedure as the one described elsewhere. ${ }^{10}$ Thermal quenching was performed by heating the samples at $500^{\circ} \mathrm{C}$ for $\sim 2 \mathrm{~h}$ and then dropping them onto a copper block at room temperature $\left(15^{\circ} \mathrm{C}\right)$. Annealing treatments were carried out in a standard furnace with temperature control in the range $\pm 5^{\circ}$.

The EPR data were obtained at $15^{\circ} \mathrm{C}$ using a conventional reflection-type $\mathrm{x}$-band spectrometer with $100 \mathrm{kHz}$ field modulation. A cylindrical cavity operating in the $\mathrm{TE}_{011}$ mode was employed. In order to perform the annealing treatments in the EPR cavity, a Varian temperature controller was employed. Crystal orientation was achieved using a goniometer, which allowed rotation of the sample in two perpendicular planes.
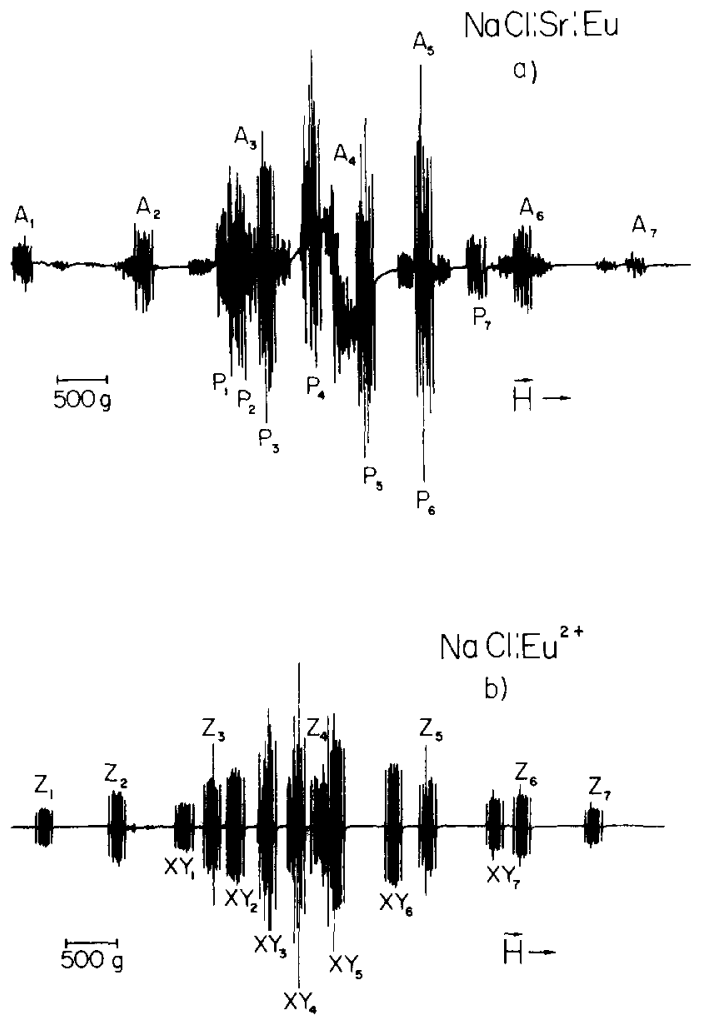

FIG. 1. (a) The axial EPR spectrum observed at $15^{\circ} \mathrm{C}$ for a fast quenched europium-strontium-doped NaCl crystal with the magnetic field along [001]. The groups labeled $A$ and $P$ have $\mathrm{H}$ oriented at the polar angles $\theta=5^{\circ}$ and $\theta=86.46^{\circ}$, respectively. (b) The orthorhombic EPR spectrum observed at $15^{\circ} \mathrm{C}$ for a fast quenched europium-doped $\mathrm{NaCl}$ crystal with $\mathbf{H} \|[001]$. The groups labeled $z$ and $x y$ have $H$ oriented at the polar angles $\theta=0^{\circ}, \phi=-\quad$ and $\theta=90^{\circ}, \phi=45^{\circ}$, respectively.

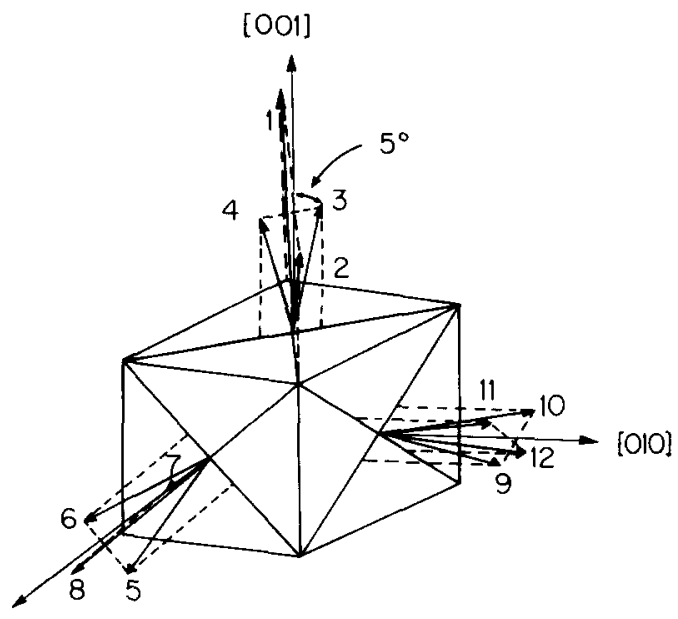

[100]

FIG. 2. Orientations of the $z$ symmetry axis of the defect responsible for the axial spectrum. They lie in $\{110\}$ planes and make angles of $5^{\circ}$ with $(110)$ directions.

\section{RESULTS AND DISCUSSION}

\section{A. Quenched samples}

Figure 1(a) shows the observed EPR spectrum of a doubly doped europium-strontium- $\mathrm{NaCl}$ single crystal after a fast quench from $500^{\circ} \mathrm{C}$ with the magnetic field applied along the [001] direction and for a sample temperature of $15^{\circ} \mathrm{C}$. Since the electronic spin of divalent europium is $7 / 2$, there are two contributions-one labeled $A$ and the other $P$ to the spectrum with $\mathrm{H} \|[001]$. These spectra are produced by distortions from the normal cubic environment. Each spectrum is made up of a superposition of several spectra which are identical except that they have different sets of equivalent principal axes. The axial symmetry due to $\mathrm{Eu}^{2+}$ in 12 inequivalent sites was identified from an angular variation of the magnetic field in the (100) and (110) planes. The maximum total spread of the spectrum occurs when the magnetic field is applied at an angle of $(5 \pm 1)^{\circ}$, with respect to the [001] direction in the (110) plane. Therefore, this direction was chosen as the $z$ symmetry axis of the complex responsible for the observed spectra. Figure 2 shows the 12 possible crystallographic directions which make angles of $5^{\circ}$ with $\langle 001\rangle$ axes in $\{110\}$ planes. If the magnetic field $\left(\mathrm{H}_{0}\right)$ is applied along the [001] direction, orientations $1,2,3$, and 4 are equivalent, i. e., all form $5^{\circ}$ with respect to the [001] direction and give the $A$ contribution to the spectrum shown in Fig. 1(a). On the other hand, the $P$ contribution is due to the equivalence between the other eight possible orientations which make an angle of $86.46^{\circ}$ with the $[001]$ direction. When the magnetic field is rotated in the $(1 \overline{10})$ plane, orientations 3 and 4 remain equivalent. However, the angles between $\mathrm{H}_{0}$ and orientations 1 and 2 become different from each other and from that for orientations 3 and 4 . This results in each $A$ line splitting into three components as $\mathrm{H}_{0}$ is rotated in the (15) plane. On the other hand, a rotation of $\mathrm{H}_{0}$ in this plane also causes each $P$ line to split into four lines, each being doubly degenerate. Finally, when $\mathbf{H}_{0}$ is 
TABLE 1. Values of the spin-Hamiltonian parameters of $\mathbf{E u}^{2+}$ in europium-strontium-doped NaCl. All crystal field parameters are given in units of G. The numbers in parentheses are the errors in the last significant digits.

\begin{tabular}{|c|c|c|c|c|c|c|c|c|}
\hline $\begin{array}{l}\text { Tupe of } \\
\text { spectrum }\end{array}$ & $\begin{array}{c}T \\
\left({ }^{\circ} \mathrm{C}\right)\end{array}$ & $g$ & $b_{2}^{0}$ & $b_{4}^{0}$ & $b_{4}^{4}$ & $151_{A}$ & $153_{A}$ & Reference \\
\hline Axial & 15 & $1.996(2)$ & $+496(3)$ & $+7(1)$ & $+60(5)$ & $-33.2(5)$ & $-14.7(5)$ & This work \\
\hline Cubic $C_{1}$ & 15 & $1.994(2)$ & $\cdots$ & $-10.0(3)$ & $\ldots$ & $-34.0(5)$ & $-15.4(5)$ & This work \\
\hline Cubic $C_{2}$ & 15 & $1.994(2)$ & $\ldots$ & $17.5(5)$ & $\ldots$ & $\cdots$ & $\cdots$ & This work \\
\hline Cubic $C_{3}$ & 15 & $1.994(2)$ & $\cdots$ & $16.4(5)$ & $\cdots$ & $\cdots$ & $\cdots$ & This work \\
\hline $\begin{array}{l}\mathrm{SrCl}_{2}: \mathrm{Eu}^{2+} \\
\quad \text { (crystal) }\end{array}$ & 24 & $1.9928(5)$ & $\cdots$ & $-15.95(12)$ & $\cdots$ & $\cdots$ & $-15.2(2)$ & 17 \\
\hline $\begin{array}{l}\mathrm{SrCl}_{2}: \mathrm{Eu}^{2+} \\
\quad \text { (crystal) }\end{array}$ & -196 & $1.9926(5)$ & $\cdots$ & $-17.87(12)$ & $\cdots$ & $\cdots$ & $\cdots$ & 17 \\
\hline
\end{tabular}

applied along the $\langle 110\rangle$ direction, there are two orientations making an angle of $90^{\circ}$ with the magnetic field, another two at $85^{\circ}$, four at $41.6^{\circ}$, and another four at 48. $3^{\circ}$. It should be pointed out, that due to the presence of the two isotopes ${ }^{151} \mathrm{Eu}$ and ${ }^{153} \mathrm{Eu}$ both with nuclear spin $\frac{5}{2}$ and of practically equal abundance, each of the fine structure lines splits into 12 hyperfine structure lines incompletely resolved due to the superposition among them.

The observed axial spectra were fitted with the spinHamiltonian:

$$
H=g \mu \mathbf{H} \cdot \mathbf{S}+\frac{1}{3} b_{2}^{0} O_{2}^{0}+\frac{1}{60}\left(b_{4}^{0} O_{4}^{0}+b_{4}^{4} O_{4}^{4}\right)+A \mathrm{I} \cdot \mathrm{S},
$$

with $S=7 / 2$ and $I=5 / 2$. The results are given in Table I. The sixth-order parameters $b_{6}^{0}$ and $b_{6}^{4}$ were not determined. However, their magnitudes must be small, in view of the fact that the positions of the resonance lines calculated from the spin-Hamiltonian parameters given in Table I agree quite well with those experimentally determined. The positive sign of the $b_{2}^{0} \mathrm{pa}-$ rameter was determined by observing small differences in the hyperfine splitting of each electronic transition taking into account that the sign of the hyperfine structure constant for $\mathrm{Eu}^{2+}$ ions has been reported to be negative. ${ }^{11}$

It should be noticed that the orthorhombic spectrum $\mathrm{III}_{1}$ due to $\mathrm{Eu}^{2+}$ ions associated with a first neighbor cation vacancy ( $\mathrm{I}-\mathrm{V}$ dipoles) was also observed in all the doubly-doped crystals investigated. Figure 1(b) shows this spectrum for $\mathrm{Eu}^{2+}$ in $\mathrm{NaCl}$ for the sake of comparison with that observed in the doubly doped samples. The origin of this spectrum has been previously described in considerable detail by several workers ${ }^{12-14}$ and it will not be discussed here for the sake of brevity.

In order to get a deeper insight into the nature of the complex responsible for the axial EPR spectrum, the optical absorption and emission spectra of a fast quenched doubly doped crystal were analyzed. It was found that the absorption spectrum consisted of the two characteristic europium broadbands in the UV range: which are due to transitions from the $4 f^{7}\left({ }^{8} S_{7 / 2}\right)$ ground state of the $\mathrm{Eu}^{2+}$ ion to states in the $4 f^{6} 5 d$ configuration. The $10 D q$ splitting measured from this spectrum was found to be quite similar, within experimental error, to that previously reported by Hernandez et al. ${ }^{15}$ for the absorption spectrum of the $\mathrm{Eu}^{2+}$ cation vacancy complexes in $\mathrm{NaCl}$. Also, the emission spectrum which consists of only one band peaking at $427 \mathrm{~nm}$ is similar to that reported for the latter complexes. ${ }^{16}$ These results suggest that in the defect responsible for the axial spectrum, the crystal field at the $\mathrm{Eu}^{2+}$ site is nearly identical to that at the site occupied by the impurity when it is dispersed into the lattice forming (I-V) dipoles. Also, taking into account that the values for the hyperfine structure constants of both ${ }^{153} \mathrm{Eu}$ and ${ }^{151} \mathrm{Eu}$ for the complex responsible for the axial spectra are the same, within quoted errors, as those previously reported by Aguilar et al. ${ }^{12}$ for $\mathrm{Eu}^{2+}$ ions associated with a nearest neighbor cation vacancy in $\mathrm{NaCl}$, in which the $\mathrm{Eu}^{2+}$ ion is surrounded by six $\mathrm{Cl}^{-}$ions, it is suggested that in the former the europium ions are principally surrounded by chlorine ions. Since the axial EPR spectrum is observed after a fast quench from high temperatures, one is tempted to consider that the complex responsible for this new spectrum is a simple aggregate involving europium and strontium ions and cation vacancies. While the simplest aggregates which can be considered are a dimer, or a trimer, the most common models for these aggregates do not have the appropriate symmetry to explain the observed axial EPR spectra reported above. Therefore, we suggest that a model of the complex responsible for the axial spectrum may be the one given in Fig. 3. This model explains the deviation of

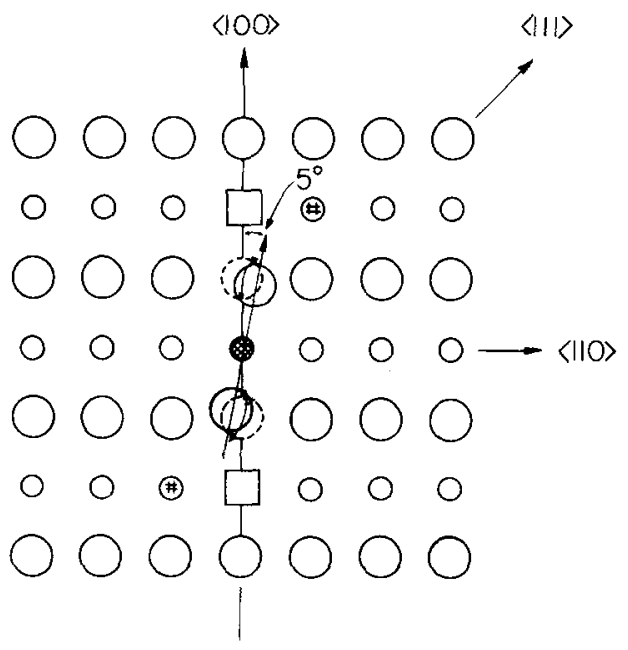

FIG. 3. Schematic model for the complex responsible for the axial spectrum involving $\mathrm{Eu}^{2+}$ and $\mathrm{Sr}^{2+}$ ions and cation vacancies. The defect axis makes an angle $\theta=5^{\circ}$ with a $\langle 100\rangle$ direction in a $\{110\}$ plane. 
the $z$ symmetry axis of the complex, with respect to the $\langle 100\rangle$ direction considering that the first neighbor $\mathrm{Cl}^{-}$ ion of the $\mathrm{Eu}^{2+}$ in the (110) plane may suffer the displacements shown in the figure, due to the electrostatic forces acting on them. The possibility that the complex may also involve more strontium ions and cation vacancies in $\langle 110\rangle$ directions can not be discarded. Evidence, preferably in the form of an ENDOR experiment is, however, required to firmly establish the model.

\section{B. Annealing treatments}

The evolution of the intensity of the axial EPR spectrum reported above has been determined as a function of the temperature in the range $25-200^{\circ} \mathrm{C}$. The results are shown in Fig. 4(a). As can be seen from this figure, no significant variation was observed in the EPR signal intensity up to $\sim 60^{\circ} \mathrm{C}$. However, on increasing the temperature, the intensity decreases and the axial spectrum wholly disappears at $\sim 120^{\circ} \mathrm{C}$. During all this annealing treatment, the intensity of the orthorhombic spectrum $\mathrm{II}_{1}$ due to $\mathrm{Eu}^{2+}$ cation vacancy dipoles also decreases. To make these measurements the sample was heated "in situ" in the EPR cavity for $10 \mathrm{~min}$. at the temperatures shown in the figure. Then, the EPR spectrum was recorded at the annealing temperature to determine its intensity. To do that, care was taken to use the same operating conditions of the EPR spectrometer. It should be pointed out that neither the axial

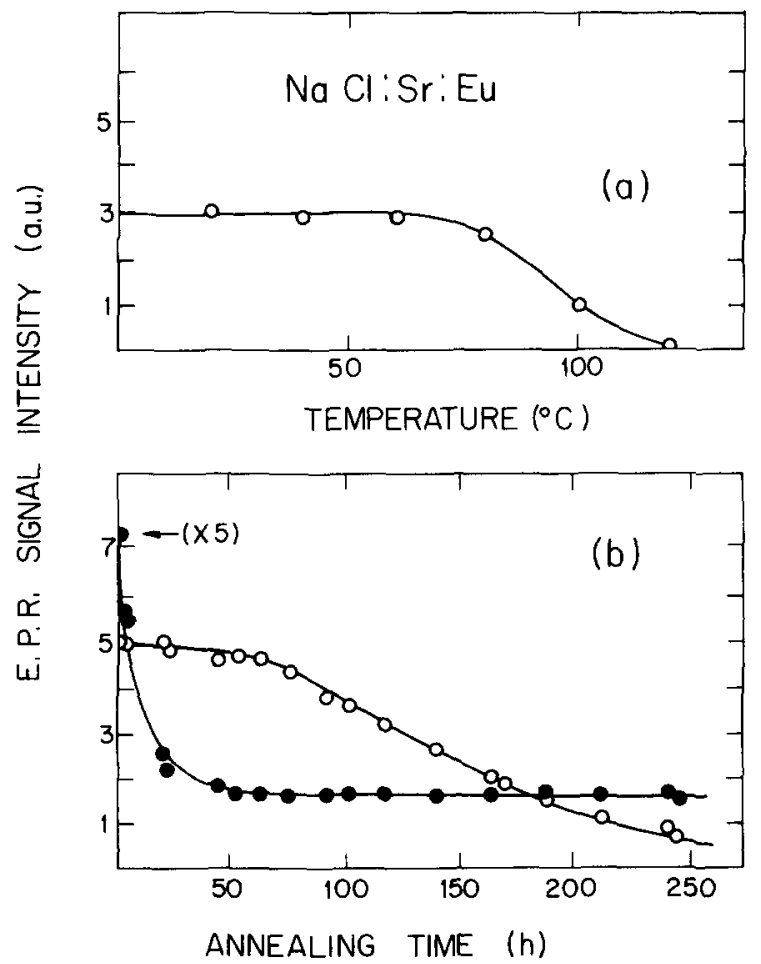

FIG. 4. (a) Evolution of the intensity of the axial EPR spectrum as a function of the annealing temperature. (b) Evolution of the intensity of the axial EPR spectrum as a function of the time elapsed at $15^{\circ} \mathrm{C}$ after a fast quench from $500^{\circ} \mathrm{C}(\mathrm{O})$; evolution of the intensity of the orthorhombic spectrum $\mathrm{II}_{1}$ due to $\mathrm{Eu}^{2+}$ cation vacancy dipoles (๑).

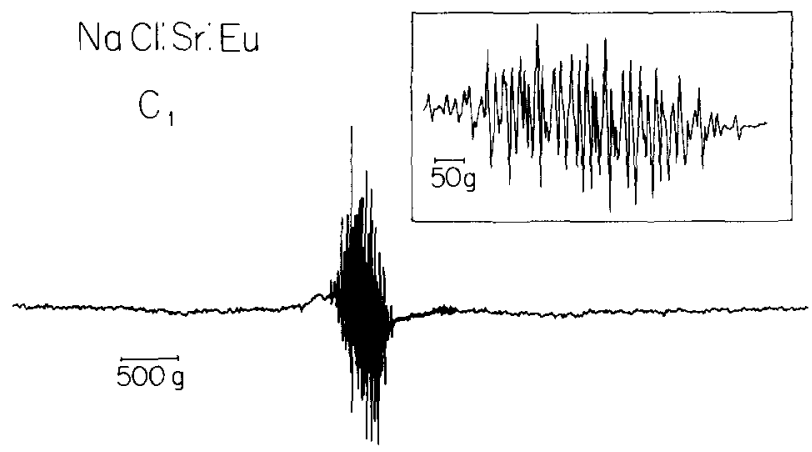

FIG. 5. The cubic EPR spectrum $C_{1}$ of an europium-strontiumdoped $\mathrm{NaCl}$ crystal which was aged about two months at $15^{\circ} \mathrm{C}$ after a fast quench from $500^{\circ} \mathrm{C}$. The magnetic field is applied along [001]. The inset in this figure shows a high resolution presentation of spectrum $C_{1}$.

EPR spectrum nor the orthorhombic $\mathrm{II}_{1}$ were recovered after the sample was heated up to $\sim 200^{\circ} \mathrm{C}$ and then fast cooled to room temperature. This result suggests that during the annealing treatment the complexes responsible for the axial spectrum form aggregates or even a second-phase precipitate in the $\mathrm{NaCl}$ matrix.

The evolution of the intensity of the axial spectrum was also studied as a function of the time elapsed at $15^{\circ} \mathrm{C}$ after a fast quench from $500^{\circ} \mathrm{C}$. Figure $4(\mathrm{~b})$ shows the quantitative results of these measurements along with the decay in intensity of the orthorhombic spectrum III $_{1}$. As is shown in this figure, the initial $\mathrm{Eu}^{2+}$ cation vacancy dipole concentration decreased by $\sim 70 \%$ in $25 \mathrm{~h}$ after the fast quench. Then, a plateau is observed up to $\sim 250 \mathrm{~h}$. An interesting result of this measurement is that the decay in intensity of spectrum $\mathrm{III}_{1}$ is much faster in the doubly-doped sample than in the single europium-doped $\mathrm{NaCl}$ crystal for the same europium concentration and experimental conditions. In fact, the dipole decay in the latter samples is $~ 3 \%$ after three months at $15^{\circ} \mathrm{C}$. On the other hand, the intensity of the axial spectrum remains essentially constant during the first $20 \mathrm{~h}$ and then begins to decay. After $250 \mathrm{~h}$, have elapsed the axial spectrum has almost disappeared and a new spectrum begins to appear. This spectrum $\left(C_{1}\right)$ is shown in Fig. 5 for a sample which was aged at $15^{\circ} \mathrm{C}$ for $\sim 2$ months. The spectrum was studied by observing its behavior as the crystal was rotated in either a (100) or a (110) plane. The maximum spread occurred when the magnetic field was applied along a $\langle 100\rangle$ direction. When the crystal was rotated in the (100) plane and the angle $\theta$ between the magnetic field and the [001] direction was approximately either $31^{\circ}$ or $58^{\circ}$, the spectrum was simplified and consisted of 12 hyperfine lines. This angular variation is the one expected from an $S$ rare earth impurity ion in a cubic crystalline field. Therefore, the observed spectrum was fitted with the spin-Hamiltonian

$H=g \mu \mathrm{H} \cdot \mathrm{S}+\frac{b_{4}^{0}}{60}\left(O_{4}^{0}+5 O_{4}^{4}\right)+\frac{b_{6}^{0}}{1260}\left(O_{8}^{0}-21 O_{8}^{4}\right)+A \mathrm{I} \cdot \mathrm{S}$,

with $S=7 / 2$ and $I=5 / 2$. The spin-Hamiltonian parameters were determined from the spectrum corresponding 


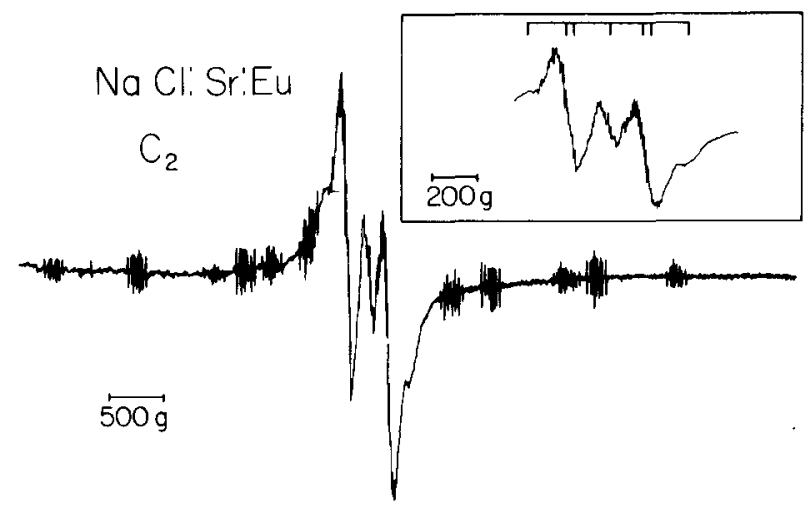

FIG. 6. The cubic EPR spectrum $C_{2}$ of an europium-strontium-doped $\mathrm{NaCl}$ crystal which was aged for five days at $200^{\circ} \mathrm{C}$ after a fast quench from $500^{\circ} \mathrm{C}$. The magnetic field is applied along [001]. The inset in this figure shows a high resolution presentation of spectrum $C_{2}$.

to $\mathrm{H} \|[001]$ and the results are given in Table $\mathrm{I}$. The magnitude of the $b_{6}^{0}$ parameter was found to be equal to zero within experimental error. The negative sign of $b_{4}^{0}$ was determined by observing small differences in the hyperfine splitting of each electronic transition.

Figure 6 shows the EPR spectrum $C_{2}$ taken on a sample which was aged at $200^{\circ} \mathrm{C}$ for five days. As can be seen from this figure, in addition to the orthorhombic spectrum $\mathrm{III}_{1}$ due to $(\mathrm{I}-\mathrm{V})$ dipoles still in solution, another spectrum consisting of seven broad lines $(\sim 40 \mathrm{G})$ is also clearly observed. The maximum splitting of this spectrum also occurs when the magnetic field is applied along a $\langle 100\rangle$ crystallographic axis and its angular behavior when $H_{0}$ was rotated in either a (100) or a (110) plane was similar to that previously described for spectrum $C_{1}$. Therefore, it was fitted with the cubic spinHamiltonian [Eq. (2)] and the results are given in Table I. The parameter $b_{4}^{0}$ was determined from measurements of line positions with the applied magnetic field parallel to a [100] axis. The sixth-order parameter $b_{8}^{0}$ was assumed to be very small and was not determined. A spectrum similar to $C_{2}$ was observed in the "as-grown" samples. However, the magnitude of the $b_{1}^{0}$ parameter corresponding to this spectrum $\left(C_{3}\right)$ is smaller than that obtained for spectrum $C_{2}$ as it is shown in Table I. Also, the widths of the observed EPR lines $(\sim 30 \mathrm{G})$ are smaller.

Previous $\mathrm{x}$-ray diffraction and electron microscopy analyses ${ }^{6,7}$ performed in strontium-doped $\mathrm{NaCl}$ have revealed the presence of $\mathrm{SrCl}_{2}$ precipitates in slowly cooled samples, as well as in samples which have been aged at temperatures in the range $\left(100-300^{\circ} \mathrm{C}\right)$. Taking advantage of this information, it is possible to conclude, from the EPR data presented above, that both spectra $C_{2}$ and $C_{3}$ are due to $\mathrm{Eu}^{2+}$ ions substituting the cation host $\mathrm{Sr}^{2+}$ in the precipitated dihalide phase $\mathrm{SrCl}_{2}$. Also, our EPR data are in agreement with the investigations mentioned above in the sense that the crystal lattice of the second-phase $\mathrm{SrCl}_{2}$ precipitate is parallel to that of the $\mathrm{NaCl}$ matrix.
Taking into account that for $\mathrm{Eu}^{2+}$ ions in cubic symmetry sites of $\mathrm{SrCl}_{2}$, the spin-Hamiltonian parameter $b_{8}^{0}$ has been determined previously ${ }^{17}$ to be very small in comparison with the $b_{4}^{0}$ parameter, it is possible to make a meaningful comparison between spectra $C_{2}$ and $\mathrm{C}_{3}$ and that reported for $\mathrm{Eu}^{2+}$ in $\mathrm{SrCl}_{2}$ single crystals. Values for the spin-Hamiltonian parameters for the latter system are also given in Table I for the sake of comparison. It is concluded that the magnitudes of $b_{4}^{0}$ for both spectra $C_{2}$ and $C_{3}$ are bigger than that for the $\mathrm{SrCl}_{2}$ single crystal by $\sim 9 \%$ and $3 \%$, respectively. This increase in the magnitude of the $b_{4}^{0}$ parameter may be due to a particle size effect of the precipitated crystals of $\mathrm{SrCl}_{2}$ in the host $\mathrm{NaCl}$. The smaller value of $b_{4}^{0}$ for spectrum $C_{3}$ compared to that of spectrum $C_{2}$ may indicate that the average size of the $\mathrm{SrCl}_{2}$ precipitates in the as-grown samples is larger than the one in the aged crystals at $200^{\circ} \mathrm{C}$. On the other hand, the large widths of the observed EPR lines for both spectra $C_{2}$ and $C_{3}$ may be the result of a size distribution of the precipitated particles of $\mathrm{SrCl}_{2}$ in the samples analyzed. However, the smaller widths of the EPR lines in spectrum $C_{3}$ as compared to those observed in spectrum $C_{2}$, suggest that the size of the $\mathrm{SrCl}_{2}$ crystals in the as-grown samples is more uniform than in the aged ones at $200^{\circ} \mathrm{C}$. It should be mentioned that similar size effects as those mentioned above have been found in the EPR spectrum of small particle crystals of gadolinium-doped $\mathrm{SrCl}_{2} .{ }^{5}$ In particular, the increase in the magnitude of the $b_{4}^{0}$ parameter has been interpreted as due to a diminution in the host lattice parameter as a consequence of the small particle size.

A comparison between the $b_{4}^{0}$ values of spectra $C_{1}$ and $C_{2}\left(C_{3}\right)$ indicates that the crystal field at the site occupied by the $\mathrm{Eu}^{2+}$ ion in the second-phase precipitate which is formed by the annealing of the quenched samples at room temperature and responsible for spectrum $C_{1}$ is smaller than at the site occupied by the impurity in the $\mathrm{SrCl}_{2}$ single crystal. As far as we know, the structure of the former precipitate has not been previously determined. However, some of its characteristics may be inferred from the results presented in this paper. First, since the sign of the $b_{4}^{0}$ parameter is similar to that for $\mathrm{Eu}^{2+}$ in $\mathrm{SrCl}_{2}$, it is suggested that the $\mathrm{Eu}^{2+}$ ion substitutes for a $\mathrm{Sr}^{2+}$ in this precipitate the coordination being the same as in the strontium chloride host, i. e., eightfold. Second, an estimation of the $\mathrm{Eu}(\mathrm{Sr})-\mathrm{Cl}$ interaction distance $\left(R_{C_{1}}\right)$ in this precipitate can be made using the results of Hurren et al. ${ }^{18}$ who established that the cubic spinHamiltonian parameter $b_{4}^{0}$ is proportional to $R^{-8.8}$, where $R$ is the interaction distance between the Eu impurity and the surrounding ligands. From this fact, we arrived at the conclusion that

$$
\frac{\left(b_{4}^{0}\right)_{\mathrm{SrCl}_{2}}}{\left(b_{4}^{0}\right)_{\mathrm{C}_{1}}}=\left[\frac{R_{\mathrm{C}_{1}}}{R_{\mathrm{SrCl}_{2}}}\right]^{8.8} \text {. }
$$

Using the values $\left(b_{4}^{0}\right)_{\mathrm{SrCl}_{2}}=-16.0 \mathrm{G},\left(b_{4}^{0}\right)_{\mathrm{Cl}_{1}}=-10.0 \mathrm{G}$, and $R_{\mathrm{SrCl}_{2}}=3.021 \AA$, it is found that $\left(R_{\mathrm{C}_{1}}\right) \cong 3.187 \AA$. It should be pointed out that in order to make this calculation, we assumed that the substitution of the $\mathrm{Eu}^{2+}$ 
ion in place of strontium does not produce any significant distortion of the $\mathrm{SrCl}_{2}$ matrix around the paramagnetic impurity. This assumption appears to be an adequate one, in view of the fact that the ionic radius of $\mathrm{Eu}^{2+}(1.12 \AA)$ is quite similar to that of $\mathrm{Sr}^{2+}(1.20 \AA)$ and therefore, a very small local distortion is expected on this basis. A similar hypothesis has been used by Newman ${ }^{19}$ to analyze the spin-Hamiltonian parameters of $\mathrm{Eu}^{2+}$ in fluorite-type crystals. Finally, from the angular variation of spectrum $C_{1}$, it is inferred that the crystal lattice of this precipitate is parallel to that of the $\mathrm{NaCl}$ matrix. It will be very interesting to perform $\mathrm{x}$-ray diffraction and electron microscopy analyses on this precipitate, which is formed by the annealing of the quenched samples at room temperature, to confirm the values suggested above.

\section{CONCLUSIONS}

In the present investigation, it has been shown that the divalent europium ion is a good paramagnetic probe to study the precipitation processes of divalent cation impurities in the host $\mathrm{NaCl}$. In particular, the data presented in this paper reveal that in addition to the stable dihalide phase $\mathrm{SrCl}_{2}$, another precipitated structure is formed by the annealing of the quenched samples at room temperature. Some of the characteristics of this second-phase precipitate were inferred from the EPR data obtained in this work. On the other hand, an interesting result of this investigation is that the magnitude of the fourth-order spin-Hamiltonian parameter describing the cubic spectrum of $\mathrm{Eu}^{2+}$ in the precipitated phase of $\mathrm{SrCl}_{2}$ appears to be dependent on the size of the precipitate which has been formed in the matrix of $\mathrm{NaCl}$.

${ }^{1}$ J. S. Cook and J. S. Dryden, Proc. Phys. Soc. London 80 , 479 (1962); Aust. J. Phys. 13, 260 (1960).

${ }^{2}$ S. Unger and M. M. Perlman, Phys. Rev. B 10, 3692 (1974).

${ }^{3}$ S. Unger and M. M. Perlman, Phys. Rev. B 12, 809, 5997 (1975); 15, 4105 (1977).

${ }^{4}$ M. Ikeya and J. H. Crawford, Jr., Phys. Status Solidi B 58, 643 (1973).

${ }^{5}$ M. Rappaz, A. Chatelain, and L. A. Boatner, J. Phys. (Paris) 7, C2-105 (1977).

${ }^{6}$ A. I. Sors and E. Lilley, Phys. Status Solidi A 32, 533 (1975).

${ }^{7}$ E. Laredo and E. Dartýge, Ack Cryst. A 27, 353 (1971).

${ }^{8}$ I. V. K. Bhagavan Raju, H. Strecker, and H. Strunk, J. Phys. (Paris) 41, C6-376 (1980).

${ }^{9}$ R. A. Cooley and D. M. Yost, Inorg. Synth. 2, 71 (1946).

${ }^{10} \mathrm{~J}$. Hernàndez A., W. K. Cory, and J. Rubio O., Jpn. J. Appl. Phys. 18, 533 (1979).

${ }^{11} \mathrm{~J}$. M. Baker and F. I. B. Williams, Proc. R. Soc. London Ser. A 267, 283 (1962).

${ }^{12}$ G. Aguilar S., E. Muñoz P., H. Murrieta S., L. A. Boatner, and R. W. Reynolds, J. Chem. Phys. 60, 4665 (1974).

${ }^{13}$ G. Aguilar S., H. Murrieta S., J. Rubio O., and E. Muñoz P., J. Chem. Phys. 62, 1197 (1975).

${ }^{14}$ J. Rubio O., H. Murrieta S., E. Muñoz P., J. L. Boldú O., and G. Aguilar S., J. Chem. Phys. 63, 4222 (1975).

${ }^{15} \mathrm{~J}$. Hernández A., F.J. López, H. Murrieta S., and J. Rubio O., J. Phys. Soc. Jpn. 50, 225 (1981).

${ }^{16} \mathrm{~F}$. J. López, H. Murrieta S., J. Hernández A., and J. Rubio O., Phys. Rev. B 22, 6428 (1980).

${ }^{17}$ R. W. Reynolds, L. A. Boatner, and M. M. Abraham, J. Chem. Phys. 52, 3851 (1970).

${ }^{18}$ W. R. Hurren, H. M. Nelson, E. G. Larson, and J. H. Gardner, Phys. Rev. 185, 624 (1969).

${ }^{19}$ D. J. Newman, Aust J. Phys, 29, 263 (1976). 